\title{
"Do Users Do What They Think They Do?"- A Comparative Study of User Perceived and Actual Information Searching Behaviour in the National Electronic Library of Infection
}

\author{
Anjana Roy ${ }^{1,2}$, Patty Kostkova ${ }^{1}$, Mike Catchpole ${ }^{2}$, and Ewart Carson ${ }^{1}$ \\ ${ }^{1}$ City ehealth Research Centre and Centre for Health Informatics, City University, \\ Northamption Square, London UK \\ ${ }^{2}$ Health Protection Agency, 61 Colindale Avenue, London, NW9 5EQ \\ Anjana.Roy@hpa.org.uk, patty@soi.city.ac.uk, \\ Mike.Catchpole@hpa.org.uk, e.r.carsonesoi.city.ac.uk
}

\begin{abstract}
In the last decade, the Internet has profoundly changed the delivery of healthcare. Medical websites for professionals and patients are playing an increasingly important role in providing the latest evidence-based knowledge for professionals, facilitating virtual patient support groups, and providing an invaluable information source for patients. Information seeking is the key user activity on the Internet. However, the discrepancy between what information is available and what the user is able to find has a profound effect on user satisfaction. The UK National electronic Library of Infection (NeLI, www.neli.org.uk) and its subsidiary projects provide a single-access portal for quality-appraised evidence in infectious diseases. We use this national portal, as test-bed for investigating our research questions. In this paper, we investigate actual and perceived user navigation behaviour that reveals important information about user perceptions and actions, in searching for information. Our results show: (i) all users were able to access information they were seeking; (ii) broadly, there is an agreement between "reported" behaviour (from questionnaires) and "observed" behaviour (from web logs), although some important differences were identified; (iii) both browsing and searching were equally used to answer specific questions and (iv) the preferred route for browsing for data on the NeLI website was to enter via the "Top Ten Topics" menu option. These findings provide important insights into how to improve user experience and satisfaction with health information websites.
\end{abstract}

Keywords: Digital Library, User Perceived and Actual Behaviour, Evaluation.

\section{Introduction}

Over the last decade, the Internet has become a ubiquitous medium essential for many activities in our daily lives. The healthcare sector has been experiencing a radical transformation in recent years as a result of this unprecedented growth of information technology. The internet has created new opportunities for better and more timely 

delivery of healthcare, but also brought challenging issues and different responsibilities for medical information providers [1].

Recent researches have investigated whether users have been able to locate information on a web site and are satisfied with content quality rather than investigating user web information seeking behaviour and satisfaction with the ease of location of information. This latter aspect of information delivery is particularly important in the case of healthcare web sites, when information is often being sought on topics that are beyond the everyday experience of the seeker.

Combining qualitative and quantitative methods provides an opportunity to understand the relationship between user perceived and actual navigation behaviour and how that impacts on the user success and satisfaction in information retrieval. We have used this approach with respect to the users of the National electronic Library of Infection (wWw.neli.org.uk) - a UK national portal for professionals in infection and public health that we are responsible for developing. The results reveal funda- mental misunderstanding of web navigation terminology and unexpected differences between user perceived satisfaction and success / failure of navigation.

\section{1 'User Perceived' versus User Actual Information 'Searching Behaviour'}

While there are a number of definitions of user information seeking and searching behaviour, for our purposes we shall use the term information searching behaviour as defined by T. Wilson, as all user activity on the web site with the purpose of finding certain information, as opposed to "surfing" the web site without a prior information need [2]. Essential indicators of the probability of knowledge discovery and overall user satisfaction and site usage are determined by (i) whether users use the site the way the site designers expect; (ii) whether they understand terminology used for site searching and (iii) how they navigate the site to find what they are looking for. This is of particular concern in the healthcare domain since failure to locate relevant information, or worse the location and use of out of date or poor quality information can have serious health consequences. Navigation can be measured by so called "disorientation" [3] - loss of a sense of orientation in relation to the web site space. Disorientation can be caused by complexity of the site navigation (browsing and searching access points), unclear terminology, and poor knowledge of the domain [4]. We measured user disorientation by their perceived satisfaction with the: (i) success in locating the most recent information; (ii) ease with which they located the information they were seeking; and (iii) general understanding of the site navigation terminology. The research questions addressed were:

1. Was there any difference between perceived (reported) user information searching behaviour and the actual (observed) behaviour?

2. To find the information, did users seek the information by browsing, searching or combined methods?

3. Did users have insight into the navigation methods they use to locate information

4. Did users find the clinical information they were looking for?

5. Is there any information searching behaviour pattern typical of certain types of users. 


\section{NeLI Navigation Structure}

NeLI (http://www.neli.org.uk ), is one of the Specialist Libraries of the National Library of Health (NLH), providing the best available evidence for clinicians, communicable disease control nurses, environmental health officers, public health specialists and others around prevention, treatment, investigation and management of infectious diseases. The information is available through the NeLI portal using two major navigation methods for Information Searching.

Browsing includes the following: (i) comprehensive $\mathrm{A}$ to $\mathrm{Z}$ listing of diseases/organism - based on MESH keywords including diseases, organisms and symptoms; or a filtered browse restricted to (ii) Top 10 Topics based on commonly accessed topics of the website 2001 - 2004; (iii) Factsheets ; (iv) Guidelines; (v) Antimicrobials \& Antimicrobial Resistance or (vi) List of Infectious Disease Society Websites

Searching: Drop-down key word search: MESH keyword-based menu. The dropdown search menu is based on the most-frequently used MESH keywords around infection and public health including Diseases, Organism, and Symptoms. As MESH does not include a taxonomy for management of disease from a public health perspective, it has been extended to cover the appropriate terms. All NeLI pages are mapped to these terms to enable precise knowledge retrieval.

Free-text search: In addition, free text search is supported to allow searching for any keywords - in this case, full text search, powered by Free Find, is used to retrieve appropriate pages.

\section{Information Searching Investigation Methods}

We developed an evaluation methodology of the NeLI users, to investigate (i) their information searching behaviour, (ii) perceived and actual behaviour; (iii) satisfaction with the information on the site; and (iv) understanding users insight into the navigation methods used by them.

\section{Study Design}

An on-line questionnaire survey and weblog analyses were combined to determine actual and perceived user behaviour. IP addresses of respondents were used to track their navigational pathway. The pathway answers from the questionnaire were compared with their weblogs.

Users were asked to have a clinical or non-clinical question in mind before navigating the site. This was essential to evaluate user information searching as opposed to surfing. The target user group were NeLI professionals who regularly visit the site. The majority of the users were confident Internet users.

To overcome the limitations of questionnaire surveys and web logs when used alone, we combine these two methods to gain a deeper understanding of user informa- tion searching behaviour, satisfaction and perception. The results of the online questionnaire addressing the navigation, perception and user needs were compared with user actual behaviour from the web logs.

Online Questionnaire: Questionnaires offer an standardised means of collecting information about people's knowledge, beliefs, and attitudes. Questionnaires were 
used to test user knowledge/attitude change before and after use of the website [5], while exit questionnaires were used to investigate user satisfaction [6]. This study determines whether there was a change in knowledge after visiting the site.

Web logs: Web logs represent a recording of user behaviour while visiting a website, and provide invaluable insights into the users' actual behaviour and the time, date and type of information the user has accessed. The drawback of web logs is that while they can reveal essential information about user navigation, they cannot provide in- sight into why users behaved the way they did and how satisfied they were with their information searching results.

Combining the methods: We looked at the declared navigational pathway preference, searching , browsing, combined method etc - (from questionnaires) and the actual path followed by the users (from web logs) for 15 users.

Details of searching and browsing behaviour were analysed for evidence of correlations between types of 'user perceived' or 'reported' and 'actual preferences' or 'observed' behaviour. We also assessed the web logs for evidence of searching or browsing for information other than that declared as the primary subject of interest on the questionnaire.

\section{Study Results}

The data collection part of this study took six weeks (August - September 2004). Nineteen users took part in the study, but we were able to identify web log data for only 15 of the users.

In the questionnaire, participants were specifically asked to record the question they were attempting to answer. For each user we compared their opinion on whether (a) they had found the answer to their question and (b) how in their view they navigated the site to get the answers and compared that to the web log evidence on (i) whether they found the most appropriate information and (b) how they actually performed the information search with regard to their question. In a majority of cases the respondents answered the their specific question in the same session as while they looking for their answers, this did not provide the opportunity to determine whether questionnaire recall accuracy was affected.

\subsection{Did the Users Find the Information They Were Looking for?}

Our results indicated that all users "reported" to have found the answers to the question they specifically were attempting to address. Our 'observed' result indicate that all except one of our users did access the page of interest specified. The user whose "reported" and "observed" page did not coincide did visit "Other websites of interest" in NeLI. So it is possible that the user did locate their topic of interest via the NeLI website - but our weblog data are unable to confirm this.

\subsection{Navigation on NeLI: Browsing and Searching}

Users were asked whether they preferred browsing, searching or combined methods for accessing information on NeLI and to provide details of the types of browsing and searching employed. 
Table 1. Users' preference about the mode of navigation as answered in question one of the questionnaire. The questionnaire and web log answers were compared by calculating the kappa measure of agreement (Table 1). The measure of agreement was 0.59 , meaning moderate agreement between the answers provided in the questionnaire and actual behaviour recorded on the web-logs, with respondents more likely to report, or recall, searching activity than browsing activity.

\begin{tabular}{|c|c|c|c|}
\hline \multirow[t]{2}{*}{ Weblog } & \multicolumn{3}{|c|}{ Questionnaire } \\
\hline & Browse (\%) & Search(\%) & Combination(\%) \\
\hline Browse & 20 & 7 & 13 \\
\hline Search & 0 & 40 & 0 \\
\hline Combination & 0 & 7 & 13 \\
\hline
\end{tabular}

On the basis of observed (web lob) behaviour, both 'Browse' and 'Search' appear to be equally preferred for navigation to find the answer to the specific question addressed by the user.

\subsection{Browsing and Searching Behaviour Details}

Questionnaire responses on "Browse behaviour" indicated that more users chose to browse a 'restricted' view of content, selecting Fact sheets, Guidelines and Top Ten topics, than use the full A-Z listing and List of Infectious Diseases. However, this preference was not statistically significant. Overall, most of the users re- ported their method of navigation accurately, when compared to web logs. For "searching" the majority of users reported behaviour also coincided with their 'observed' behaviour (Table 2). A wide range of options were used in the 'pull down options' in search. These varied from searching information on specific viruses e.g. Cytomegalovirus, seeking information on travel associated illnesses to looking information for contributors. None of the users in this study used the "Free text search".

The numbers in each column do not add up to 100, because users indicated that they used a combination of options in Browse along with the search options.

These results indicate the following:

1. Users were successful, and perceived themselves to be successful, in locating the information they were seeking.

2. Most users located information using a combination of navigation options; in the majority of the cases this has been reported accurately.

3. There is no significant preference for any particular browse option (other than that 'Antimicrobial Resistance' was not used at all in this study).

4. The "Pull down" menu in the search option is the only search option used in this study - "Free-text" was not used at all. 
Table 2. Comparison of reported and observed browse and search behaviours

\begin{tabular}{|llcc|}
\hline Browse & & $\begin{array}{c}\text { Questionnaire } \\
\text { (percent) }\end{array}$ & $\begin{array}{c}\text { Web logs } \\
\text { (percent) }\end{array}$ \\
\hline & Ato Zlisting of pages on NeLI & 7 & 13 \\
& Top 10 Topics & 13 & 20 \\
& Factsheets listed on NeLI & 13 & 13 \\
& Guidelines listed on NeLI & 13 & 13 \\
& Antimicrobials Resistance & 0 & 0 \\
& List of Infectious Disease SocietyWebsites & 7 & 7 \\
& & & \\
& & 67 & 60 \\
& Pull down Menu & 0 & 0 \\
& Free Text Search & & \\
& & & \\
\end{tabular}

\subsection{Cases Where the Users Reported Navigation Behaviour and Observed Behaviour Did Not Match}

When the 'observed' and 'reported' results did not match - it was due to one of the following reasons:

1. Users were 'observed' to use a combination of navigational options while reporting only one option to access the page of interest.

2. Users reported using a different navigation technique to that observed e.g they reported to be using 'search' - but were observed to reach the page of interest via 'Browse' options.

$80 \%$ of the users reported that they found navigation of the site easy while $20 \%$ reported it to be difficult. The $20 \%$ expressing difficulty 'reported' their method of navigation correctly, i.e. their 'observed' and 'reported' behaviour matched perfectly.4.5. Navigation to "Other Pages of Interest"

The web log data were able to inform us of the information searching method used when users visited "other pages of interest" in this session - typically accessed after answering their "specific question". Web log analysis indicates that the most favoured method of navigation in this case was Top 10 Topics, which also matches our specific question web log data (Table 3). Of the users, majority (93\%) used the browsing option to visit other pages of interest, few $(7 \%)$ used the search 'Drop Down - key word search', with no-one using the Free Text Search. We have not asked users for their navigation methods for seeking information other than that used to answer their clinical question, as we did not expect such a high percentage of users to "surf" while answering the questionnaire. Therefore, we do not have the questionnaire data for comparison in this case, but did find the web log data very revealing. We can conclude that for 'surfing ' through the site Top 10 Topics is the favoured option.

Please note : The total percent of users does not add up to 100 as this table shows the total number of people who actually went to "other" pages of interest - while $19 \%$ only answered the questionnaires during the session. 
Table 3. Navigational method used to access the "other pages of interest"

\begin{tabular}{|llc|}
\hline \multirow{3}{*}{ Browse } & & Web logs (percent) \\
& A to Z listing of pages on NeLI & 7 \\
& Top 10 Topics & 27 \\
& Factsheets listed on NeLI & 13 \\
& Guidelines listed on NeLI & 7 \\
& Antimicrobials \& Antimicrobial Resistance & 7 \\
& List of Infectious Disease Society Websites & 13 \\
Search & & \\
& Drop Down - Key word search & 7 \\
& Free Text Search & \\
\hline
\end{tabular}

\section{Discussion}

Development of successful healthcare Web sites demands regular evaluation to provide better understanding of the underlying issues of user satisfaction with the provided information and ease with which it can be located. In this paper we investigated the discrepancy between user perceived and actual navigation behaviour.

In this study we have correlated the pattern of navigation of individual users with their views on the NeLI web site and analysed the discrepancies between the sets of data. Choo et al. have used questionnaires and web logs and developed a modified model to describe common repertoires of information seeking by users in general [7]. Here, we have tried to determine whether the users have reported their navigation strategy correctly - or have become disorientated, which can arise from unfamiliarity with the structure or conceptual organisation of the site. The decision regarding which web page to view next involves understanding one's current location within the site, then selecting the proper route .

The users were able to locate the information they were seeking specifically from the NeLI website. This implies that largely the users understand the terminology used for navigating the site, and that they use the site as the developers expect them to.

However, the 'user perceived' and the actual user behaviour did not always tally. There appears to be slight confusion regarding the search and browse options, with a few users using a combination of options to access the information they were seeking, but failing to realise this, i.e. they reported only using one navigation methodology. Web-space disorientation could be a likely explanation. Therefore, efforts are being made to make the browse and search access points clearer and more simple to use in the next version of the NeLI.

In this study we wanted to determine the preferred method of navigation of the NeLI site. This appeared to depend on :

1. whether the user was trying to answer a specific clinical/non-clinical question using the NeLI website. We obtained this information primarily from the web logs study, which indicated a preference for searching to locate information. When searching, only the "pull down" menu was used - free text was not used at all. Where browse was used, there was little evidence of a preferred point of en- try, although users did seem to be more likely to browse within a particular type 
of document ('Factsheet', 'Guidelines', etc) than opt for a subject-based filter as the starting point for browsing.

2. When the users were just surfing though NeLI their preference was to browse, using "Top 10 Topics" as a starting point i.e. they appeared to be content to be guided by the website authors, or other users, as to where they might find the most useful information. In this case we only had information on "observed" method of navigation. The popularity of this method of navigation has led the de- velopers to research this area further and broaden the scope of this option. Cur- rently the NeLI developers are in the process of identifying the Top Twenty Five Topics in infectious diseases.

The users found the site to be too "wordy" and suggested improvement by addition of clips, videos and images. This information is invaluable for the site developers and obviously cannot be obtained from any web log analysis. This study will enable web developers to improve navigation system within their systems.

\section{Conclusion}

By combining qualitative and quantitative methods (online questionnaires and weblogs) we could demonstrate that there is reasonable correlation between user perceived information seeking behaviour and the actual behaviour. This pilot study was part of a long-term project investigating impact evaluation of healthcare Web sites, and the development of validated tools for providing feedback that can inform web- site improvements.

\section{References}

1. Armstrong, R.: Appropriate and effective use of the Internet and databases. Clin. Rheumatol. 22(3), 173-176 (2003)

2. Wilson, T.D.: Human Information Behaviour. Information Science 3(2) (2000); special Issue on Information Science Research

3. Juvina, I.: The Impact of Link Suggestions on User Navigation and User Perception. In: Ardissono, L., Brna, P., Mitrović, A. (eds.) UM 2005. LNCS (LNAI), vol. 3538, pp. $483-$ 492. Springer, Heidelberg (2005)

4. Draper, S.W.: Supporting use, learning, and education. Journal of computer documentation 23(2), 19-24 (1999)

5. Madle, G., Kostkova, P., Mani-Saada, J., Williams, P., Weinberg, J.R.: Changing public attitudes to antimicrobial prescribing: can the Internet help? Informatics in Primary Care 12(1), 19-26 (2004)

6. Huntington, P., Williams, P., Nicholas, D.: Age and gender user differences of a touch- screen kiosk: a case study of kiosk transaction log files. Informatics in Primary Care 10(1), 3-9 (2002)

7. Choo, C.W., Detlor, B., Turnbull, D.: 2000ormation seeking on the web- An integrated Model of browsing and searching. First Monday 5(2) (February 2000), http://firstmonday.org/issues/issue5_2choo/index.html 